\title{
Escuela Militar de Aviación, memoria y género: totalidades en el recuerdo femenino, realidades fragmentadas en la evocación masculina ${ }^{1}$
}

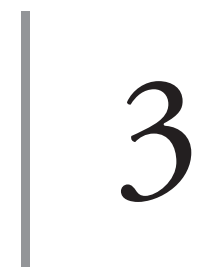

https://doi.org/10.21830/9789585380219.03

\author{
José Fernelly Dominguez Cancelado \\ Escuela Militar de Aviación "Marco Fidel Suarez"
}

Resumen. El ingreso de 34 mujeres bachilleres, junto con 94 bachilleres hombres, para integrar el primer Curso Mixto N. 73 de Oficiales de Escuela, inauguró una serie histórica dentro de la historia general de la Fuerza Aérea Colombiana (FAC), cuyo estudio forma parte de un proyecto de investigación más amplio que ha buscado comprender las características del desarrollo educativo de la Escuela Militar de Aviación "Marco Fidel Suárez" (EMAVI) en los cien años de existencia de la FAC. Para lograr este objetivo se ha construido un diseño basado en el paradigma interpretativo, el cual ha utilizado el enfoque cualitativo para analizar los datos obtenidos mediante estrategias etnográficas y de análisis documental. Uno de los hallazgos importantes del análisis de las entrevistas realizadas fue la constatación de formas diferenciadas de recuperación de la memoria en hombres y mujeres. Estas diferencias parecen estar asociadas a la cultura objetiva, masculina, y a la cultura subjetiva, femenina.

Palabras clave: educación; cultura femenina; cultura masculina; formación militar; Fuerza Aérea Colombiana; historia; memoria; mujeres militares.

1 El presente capítulo es uno de los productos del proyecto de investigación "Recuperación de la memoria histórica del proceso de formación de la Escuela Militar de Aviación "Marco Fidel Suárez", del Grupo de Investigación en Ciencias Militares Aeronáuticas y Administrativas (GICMA), con código de registro en Minciencias COL0140489. Los puntos de vista y los resultados presentados en este capítulo pertenecen al autor y no reflejan necesariamente los de las instituciones participantes.

2 Magíster en Sociología de la Universidad del Valle. Especialista en Teoría y Métodos de Investigación en Sociología y licenciado en Historia de la misma universidad. Docente de la Universidad "Antonio José Camacho", Cali, Colombia. Docente-investigador del Programa de Ciencias Militares Aeronáuticas de la Escuela Militar de Aviación "Marco Fidel Suárez" (EmAvi), Cali, Colombia. Orcid: https://orcid.org/0000-0002-2886-7576 - Contacto: jose.dominguezc@emavi.edu.co 


\section{Introducción}

Buena parte de las publicaciones realizadas sobre la historia de la Escuela Militar de Aviación "Marco Fidel Suárez" (Emavi) se han hecho más desde la perspectiva periodística que de la interpretación histórica, la cual permite matizar las realidades de cada época, pero no establecen contextos históricos para analizar el desarrollo educativo que ha dado soporte a la aviación militar en sus cien años de existencia.

Ese vacío es el que ha tratado de llenar la investigación histórica que ha precedido dos de nuestras publicaciones: Domínguez (2019a, 2019b) y el presente artículo. La investigación ha pretendido documentar la memoria del proceso educativo de la EMAVI, desde su creación en 1933 hasta el año 2018, junto a la identificación de los principales cambios y transformaciones presentados en la formación militar, aeronáutica y profesional de la Escuela Militar de Aviación "Marco Fidel Suárez".

En el desarrollo del proyecto de investigación hemos tratado de responder preguntas como las siguientes: ¿Cuáles han sido los principales cambios y transformaciones presentados en cada uno de los ámbitos de la formación en la EMAVI (militar, aeronáutico y académico-profesional)? ¿Cómo se ha configurado la memoria en este proceso de larga duración?

Para dar respuesta a estas preguntas fijamos los siguientes objetivos: comprender el proceso formativo de la EMAVI desde su creación en 1933 hasta el año 2018, como objetivo general. Para desarrollarlo, nos propusimos: describir el proceso formativo de la EMAVI; identificar los principales cambios y transformaciones que ha tenido la formación militar, aeronáutica y profesional, y documentar la metodología desarrollada para la recuperación de la memoria de la Escuela.

\section{Metodología}

El proyecto se inscribió en el paradigma hermenéutico (Gadamer, 2007), en la medida en que ha pretendido interpretar y comprender el significado de las acciones humanas y de las prácticas sociales que han tenido lugar en la Emavi. Se desarrolló una metodología de corte cualitativo con un alcance comprensivo, dentro de un proceso inductivo, que posibilita sistematizar la experiencia desarrollada por la institución. 
Asimismo, se realizó un diseño documental buscando construir datos que permitieran periodizar el desarrollo histórico de la EMAVI y se planteó un diseño etnográfico a partir de un grupo focal, de entrevistas abiertas y semiestructuradas a exintegrantes y actuales miembros de la comunidad educativa de la EMAvi.

En el campo de la historia, el proyecto trabajó con base en la concepción de la "Historia General" propuesta por Foucault (2011), en la cual no existen puntos causales únicos que expliquen el devenir histórico, sino la concurrencia de series que inician o terminan por medio de fracturas o discontinuidades.

En este sentido, los diversos cambios que la institución ha experimentado en la formación militar, aeronáutica y profesional a lo largo de sus 87 años de historia han constituido, entonces, algunas series históricas en las cuales han tenido incidencia acontecimientos tanto internacionales como nacionales, en el proceso de modernización del Estado y de la sociedad como estadio intermedio entre una sociedad tradicional, premoderna, y una hipotética sociedad moderna.

Como parte de los resultados de este estudio se describió y se analizó el contexto histórico en el cual surge y finalmente se consolida la EMAvi desde los últimos gobiernos de la Hegemonía Conservadora, pasando por los gobiernos de la República Liberal y el retorno de los conservadores al poder, hasta los gobiernos del Frente Nacional, la nueva Constitución de 1991, como máximo logro del esfuerzo por modernizar a Colombia, y el ingreso de las mujeres a la formación como Oficiales de Curso Regular, finalizando con un análisis de los procesos de recuperación de la memoria a partir de los testimonios y las declaraciones de todas las personas entrevistadas, el cual forma parte del presente capítulo.

\section{Análisis y discusión de los resultados}

En la ejecución del proyecto de investigación sobre la historia del desarrollo educativo de la EMAvi se ha tratado de comprender su origen, su desarrollo y los logros alcanzados como institución de educación superior. En función de esto se ha asumido la ardua tarea de recuperar la memoria del 
proceso educativo de la EMAVI, para lo cual se entrevistaron individualmente o en grupo focal más de cuarenta personas, quienes tienen o han tenido vínculos con la Escuela, entre docentes, oficiales activos y retirados de ambos sexos, y se analizaron ocho testimonios escritos. Las entrevistas cubren un lapso de tiempo que va desde el más antiguo, el CT(R) Marco Tulio Acosta (q. e. p. d.), Curso 15, incorporado en 1942, hasta los y las oficiales activos, con rango de Teniente Coronel del Curso 73 de 1997.

Se trabajó a partir de dos dimensiones: la dimensión cognitiva y la dimensión pragmática (Ricoeur, 2003), con las cuales se trató de responder dos preguntas: (1) ¿Cómo se recuerda a la EMAVI? (2) ¿Para qué se la recuerda?

Para contestar la primera pregunta se parte del concepto de la memoria colectiva, que Ricoeur (2003) toma de Maurice Halbwachs. Este parte del supuesto de la existencia de una memoria social que preserva el saber sobre acontecimientos, valores y relaciones dentro de un grupo, y que es la base de la conservación de su identidad. Halbwachs propone el concepto de memoria colectiva, que permite pensar la memoria y sus objetos en unidades sociales mayores. Al respecto, dice que "la memoria depende del entorno social", de los "marcos sociales" (Halbwachs, 2004b, p. 7).

En el ámbito de la cotidianidad, ¿qué y cómo se recuerda? La mayoría de los hombres entrevistados, incorporados antes del Curso 73, cuando ingresaron las mujeres, al preguntarles por sus vivencias en la EMAVI, estructuraron sus recuerdos a partir del componente militar como principal elemento que les otorga identidad: la intensidad de los ejercicios físicos, la rudeza de la instrucción militar, a la cual aceptan como un factor importante en su formación para la vida. Unos pocos se apartan de las supuestas bondades de este tipo de formación y asumieron una posición crítica abierta o de soslayo frente a ella. Por ejemplo, un General retirado hace varios años, recuerda la vida militar como muy difícil: "No nos formaban, sino que nos deformaban, los Derechos Humanos no existían. Hacían con los cadetes lo que les daba la gana” (Domínguez, 2017b).

Como ya se dijo, los recuerdos de todos los oficiales entrevistados están asociados a la vida militar, al número del curso al cual fueron incorporados y no tanto al año en que iniciaron sus estudios básicos, pues el número del 
curso es un indicador de la antigüedad como punto de comparación con los demás y como punto inicial de configuración de sus recuerdos. En sus relatos se rememoran sus dificultades, sus satisfacciones, la formación militar recibida, pero no la vida académica propiamente dicha. Con algunas excepciones. Por ejemplo, los recuerdos académicos de quienes se profesionalizaron en la década de los años 60 y 70 están asociados a los docentes de la Universidad del Valle o de la Universidad del Cauca, a donde eran enviados quienes más se destacaban para que completaran sus materias y obtuvieran un título profesional. La explicación de esta forma de recordar puede estar relacionada con el hecho de que su proceso de formación académica ya no tenía que competir con la formación militar, ni con la formación como pilotos.

Y aquí, la diferencia la hacen las mujeres oficiales del Curso 73; la mayoría de ellas sí incluyen el rendimiento académico como parte de sus recuerdos y la experiencia de haber cursado maestrías en diferentes campos del conocimiento, haber estado vinculadas a proyectos y a grupos de investigación en diversas universidades del país.

Uno de los postulados de la sociología de la memoria establece que no recordamos desde el pasado hacia el presente, sino a la inversa. Recordamos desde el presente, desde lo que somos en el presente, o, dicho de mejor manera, desde lo que al grupo o a la sociedad a la que pertenecemos le interesa, valora y percibe en el presente. Desde allí dirigimos nuestra mirada hacia el pasado. Entonces, nuestros recuerdos sobre la vida en las instituciones hablan más de lo que ellas significan para nosotros en el presente, que de lo que fueron realmente en el pasado. Sobre esto, dice Halbwachs en La memoria colectiva que nuestros recuerdos se adaptan a lo que percibimos en el presente (2004a, p. 25). Por eso cada uno de los entrevistados regresó al pasado, entre otros factores, a partir de lo que es en el presente: oficial activo o retirado.

En este sentido, podemos arriesgarnos con una hipótesis a modo de interpretación, y es la siguiente: las instituciones militares forman parte de lo que Georg Simmel (1934) caracteriza como un producto de la "cultura objetiva", construida por los hombres a lo largo de la historia de la humanidad, en la cual predominan como amos y señores hasta finales de los años 90, cuando las mujeres accedieron a la formación militar como oficiales de Escuela, en 
igualdad de condiciones, en muchos países de Europa, América Latina y los Estados Unidos, entre otros. En el caso colombiano, para los hombres entrevistados, el recuerdo de su formación militar significa retomar el punto de vista de los grupos que han pasado por la experiencia militar, que reproducen las tradiciones culturales masculinas destinadas a resaltar los valores patriarcales característicos de las historias militares: su capacidad física, el ejercicio del mando, sus éxitos militares o el estatus que otorga el uniforme, entre otros. Por ejemplo, el CT(R) Marco Tulio Acosta (q. e. p. d.), quien tenía 96 años al momento de entrevistarlo (Domínguez, 2017a), fue alférez de la Escuela Militar del Ejército y en calidad de tal, en febrero de 1942, fue enviado a la EMAvi para incorporarse al Curso 15. Sobre su experiencia enfatizaba: "Cuando salíamos los domingos por las calles de Cali, las muchachas nos admiraban, éramos llamativos".

Para el caso de las mujeres, desde que accedieron a la educación superior en 1935, la profesionalización ha funcionado como uno de los principales mecanismos de movilidad social y de reconocimiento de estatus para ellas. Por esto, en general, las narrativas del pasado están frecuentemente asociadas a sus éxitos académicos y profesionales en relación con su carrera de pregrado o a los posgrados realizados.

Otra diferencia importante entre hombres y mujeres, en relación con lo que recuerdan, es el componente familiar. Los hombres olvidaron o no asociaron sus recuerdos a sus compańeros de curso ni a las mujeres en su calidad de esposas, novias o hijas. A diferencia de las mujeres, para quienes sus compañeras, tanto las que continuaron como las que se retiraron o fallecieron (como es el caso de la TE Johana Ximena Herrera, q. e. p. d.), aparecen como parte importante de sus recuerdos, siendo el marco de su memoria el imaginario de pertenencia a la Fuerza Aérea Colombiana (FAC) como a "una gran familia”. Recuerdan quién les ayudó en los momentos de dificultad.

A propósito de esto, en sus relatos, los hombres no recuerdan o no reconocen sus debilidades durante el proceso formativo. En la mayor parte de las fotografías proporcionadas por las actuales tenientes coroneles, provenientes del Curso 73, para recordar los ańos de permanencia en la EMAVI, siempre aparecen en grupo: con sus compañeras de dormitorio o en diversas activi- 
dades, por ejemplo, en compañía de quienes realizaron su primer vuelo solo o sus salidas a "crucero". Igualmente, en la representación posterior de su vida como oficiales, la acompańaron de fotografías en las que aparecen sus esposos, sus padres o sus hijos como parte de su realización personal. Quienes no aportaron fotografías, sus esposos y/o hijos, aparecen en su relato con expresiones de gratitud.

\section{La solidaridad femenina como recurso valioso}

Cuando los grupos humanos se enfrentan al esfuerzo físico, a las adversidades o al fragor del combate en una batalla donde puede estar en riesgo la vida o la integridad física y logran resolver sus dificultades de forma asertiva, se construye entre sus integrantes una red de hilos invisibles de afecto y de solidaridad; en suma, un tejido social tan fuerte que les permite enfrentar los nuevos retos con una mayor confianza y optimismo, que ya no están afincados solo en las fortalezas o en las ventajas individuales, sino, principalmente, en las sinergias que solo se generan en los grupos muy cohesionados y solidarios. Son los amigos y las amigas de curso, los compañeros de un viaje muy difícil de enfrentar, quienes se dan ánimo en los momentos de decaimiento, de tristeza y cuando cada una sentía que ya no podía más y que iba a desertar. Pero también son los amigos con quienes se comparten las alegrías y el optimismo de poder lograr las metas. Y esta red puede perdurar toda la vida. Por esto es muy común escuchar a oficiales y exoficiales referirse a la FAC como su gran familia.

\section{Las diferencias de género}

Las respuestas a la pregunta sobre el porqué de las diferencias de género en el proceso de recuperación de la memoria podemos encontrarlas al analizar, con Simmel (1934), el proceso de configuración de dos culturas relacionadas con el género: la "cultura objetiva" o cultura masculina y la "cultura subjetiva" o cultura femenina. Al caracterizar el pensamiento masculino como "objetivo" se quiere significar que procede de forma racionalista, fragmentando la realidad en cada uno de sus componentes y abordando en cada momento solo uno de ellos. La dirección de sus procesos mentales va, desde su centro espiritual y emocional, desde su yo, hacia la periferia. Dijéramos que se despoja 
de ese centro emocional abandonándose por entero a una sola de las variables de su vida, sin mezclar sus sentimientos con la realidad que enfrenta. Dice Simmel (1934):

Dijérase que el varón puede emplear sus energías en una sola dirección fija, sin menoscabo de su personalidad. $Y$ es porque considera esa actividad diferenciada, desde un punto de vista puramente objetivo, como algo separado y distintivo de su vida personal y privada. (p. 19)

Entonces, resulta lógico suponer que, si la base de los recuerdos son las actividades realizadas "en una sola dirección", su evocación en el futuro tendrá esa dirección como línea orientadora. Si la vida diaria de los estudiantes de la EMAVI se distribuía entre la formación académica, la formación militar, la formación como pilotos y, además, la práctica deportiva, los varones las asumen como cuatro ámbitos diferenciados y la recuperación de la memoria también adopta la misma estrategia diferenciada. Como ya se dijo, son los recuerdos de la vida militar los que primero se recuperan y predominan en buena parte de la evocación.

A diferencia de esta lógica masculina, las mujeres piensan en la realidad como una totalidad. La dirección de sus procesos mentales va en sentido contrario al de los hombres, de la periferia hacia su centro emocional y mental, hacia su yo, de tal forma que emociones y realidad aparecen como un todo. Dice Simmel (1934):

En la mujer, la periferia está más estrechamente unida con el centro y las partes son más solidarias con el todo, que en la naturaleza masculina. Y así resulta que cada una de las actuaciones de la mujer pone en juego la personalidad total y no se separa el yo y sus centros sentimentales. (p. 19)

Uno de los ejemplos más evidentes sobre los resultados del ejercicio de la cultura femenina en el campo militar lo recuerda el BG Silva (Domínguez, 2018), en la cual recuerda las diferencias entre hombres y mujeres en relación con el cumplimiento de una tarea como la guardia. Los hombres atienden solamente la vigilancia del espacio asignado, mientras que las mujeres tienen en cuenta la totalidad de la base. Asumen la responsabilidad por esa totalidad y no solamente por un puesto de vigilancia. 
Pero, además, se puede considerar, a modo de hipótesis, otra explicación para este comportamiento diferencial entre hombres y mujeres. En su imaginario, consciente o inconscientemente, las mujeres asumen las unidades militares como su casa. La casa donde vive la gran familia de la FAC, es el todo donde las mujeres son motores multitarea y asumen la responsabilidad, no solo por la parte asignada, sino por el conjunto de la familia. Esto nos lleva a otra de las características de la cultura femenina: la casa, como la gran conquista de la cultura femenina para el sustento de la humanidad en su conjunto. Al respecto, Simmel (1934) afirma que "para la mujer, la casa significa la vida entera plasmada a modo doméstico" (p. 45).

De allí, una de las diferencias en la forma como los hombres y las mujeres entrevistados recuerdan el pasado: los hombres construyeron sus narrativas a partir de funciones especializadas separadas de su yo. Las mujeres recurrieron a la figura de la "gran familia de la Fuerza Aérea". Para matizar sus recuerdos, aportaron fotografías en las que aparecen como equipo, por ejemplo, en la intimidad de la habitación que fue su hogar, su casa, durante cuatro años ${ }^{3}$.

Las mujeres piensan y recuerdan a partir de la totalidad, mientras que los hombres lo hacen a partir de fragmentos de esa realidad.

\section{¿Para qué se recuerda a la EMAVi?}

Como ya se dijo, esta pregunta responde a la dimensión pragmática del problema. Corresponde a la noción ética de la memoria, a la que se considera como un problema de justicia. Se trata de reconocer el importante papel de las personas responsables de la formación de los oficiales, que ya no están, y a quienes se hace justicia mediante el recuerdo: "Al extraer de los recuerdos traumatizantes su valor ejemplar, logran transformar 'la memoria en proyecto' [...] y es este mismo proyecto de justicia el que da al deber de memoria la forma del futuro y del imperativo" (Ricoeur, 2003, p. 120).

Todos los militares entrevistados, hombres y mujeres, reconocen el papel que han tenido en su formación los oficiales a cuyo mando estuvieron y que ya no están. Atribuyen su identidad actual y las realizaciones alcanzadas a

3 Véase también nuestro análisis sobre la cultura subjetiva desarrollada por las mujeres en la Emavi (Domínguez 2019a, capítulo 3). 
la formación recibida. En este sentido, las mujeres tienden a recordar a sus formadores con nombres propios. Los hombres no los mencionan en forma individual y los cobijan en expresiones como "la institución me formó", "los altos mandos me ordenaron” y otras. En relación con la deuda con el pasado, su evocación se guía por la línea de mando, "hacia arriba”, es decir, las realizaciones, los aportes o las experiencias vividas bajo el mando de los oficiales de mayor rango militar o antigüedad. Los de menor rango o antigüedad no aparecen, en general, en sus relatos.

\section{Conclusiones}

En las formas de recuperación de la memoria social en la FAC se pueden apreciar diferencias de género. Los hombres, en general, recuerdan a partir de la racionalidad que fragmenta su entorno de vida, siendo la ruta más común de recordación la vida propiamente militar y no la experiencia académica. En esa línea de recordación, no es común que aparezcan sus compañeros claramente identificados como contribuyentes de su formación. Las mujeres que han formado parte de su vida no ocupan un lugar principal en sus recuerdos. Aparecen tangencialmente. Las mujeres recuerdan desde la totalidad del mundo que las ha rodeado: en primer lugar las relaciones de amistad y solidaridad con sus compañeros y compañeras de formación, las experiencias de la vida académica y militar, y destinan un lugar importante a recordar a sus padres, esposos e hijos como factores importantes en sus éxitos. En su imaginario, la FAC aparece como una gran familia.

Todos recuerdan a partir de las dos variables que definen su lugar en la estructura: el número del curso, que define la antigüedad y la línea de mando. Se recuerda con mayor frecuencia a quienes están por encima en estas dos variables y no a quien está debajo. Y, finalmente, se recuerda a la EMAVI y a la FAC para reconfirmar que la decisión profesional tomada fue correcta. Tienden a olvidar las experiencias desagradables y las posibles frustraciones, para atribuir a las instituciones del poder aéreo la fuente de todo lo bueno que en el presente tienen. 


\section{Referencias}

Domínguez, J. F. (2017a). Entrevista con el CT(r) Marco Tulio Acosta. Medellín, Colombia, 19 de mayo de 2017.

Domínguez, J. F. (2017b). Entrevista con el GR Edgar Lesmes Abad. Cali, Colombia: 27 de octubre de 2017.

Domínguez, J. F. (2018). Entrevista con el BG Carlos Fernando Silva Rueda. Cali, Colombia: 11 de mayo de 2018.

Domínguez, J. F. (2019a). Historia del desarrollo educativo de la Escuela Militar de Aviación "Marco Fidel Suárez": Expresión del proceso de modernización en Colombia, 19332018. Escuela Militar de Aviación.

Domínguez, J. F. (2019b). Pioneras de aire y tierra: Historia del proceso de incorporación de las primeras mujeres como Oficiales de Curso Regular en la FAC en 1997. Escuela Militar de Aviación.

Foucault, M. (2011). Arqueología del saber. Siglo XXI.

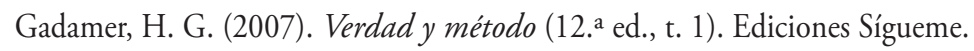

Halbwachs, M. (2004a). La memoria colectiva. Prensas Universitarias de Zaragoza.

Halbwachs, M. (2004b). Los marcos sociales de la memoria. Anthropos.

Ricoeur, P. (2003). La memoria, la historia, el olvido. Editorial Trotta.

Simmel, G. (1934). Cultura femenina. Revista de Occidente. 